In einer vor zwei Jahren publizierten Studie hatten australische Forscher keinen klinisch bedeutsamen Zusammenhang zwischen Rückenschmerzen und dem Wetter finden können. Der Aufschrei Betroffener war groß. Nun ist die Untersuchung wiederholt worden. Das Ergebnis ist das gleiche - der Aufschrei vermutlich auch.

An der Studie, geleitet von Seniorautor Daniel Steffens von der Universität Sydney, waren 981 Patienten mit einer frisch aufgetretenen Episode von lumbalen Rückenschmerzen beteiligt. Analysiert wurden die offiziellen Daten des australischen Amtes für Meteorologie, und zwar zum einen die durchschnittlichen Wetterwerte für den Tag vor Beginn der Beschwerden, zum anderen die Veränderungen der Wetterlage zwei Tage bis einen Tag vor dem Schmerzbeginn. Zur Kontrolle dienten die Zeiträume eine Woche und einen Monat vor dem kritischen Zeitfenster.

Weder für die Niederschlagssituation noch für die Luftfeuchtigkeit und auch nicht für die Windverhältnisse oder den Luftdruck war ein Zusammenhang mit dem Einsetzen von Kreuzweh festzustellen. Allenfalls gingen wärmere Temperaturen mit einer marginal höheren Schmerzhäufigkeit einher. Die Forscher halten den Befund aber für klinisch irrelevant.

\section{Ergebnis nicht auf chronische Schmerzen übertragbar}

Die Autoren um Steffens räumen ein, es habe sich bei den Probanden um Patienten mit akuten Rückenschmerzen gehandelt. Deshalb sei das Resultat nicht ohne Weiteres auf Menschen mit chronischen Schmerzen oder Arthritis übertragbar.
Deren Wetterfühligkeit zu erforschen, bleibe künftigen Studien vorbehalten.

Den Zusammenhang zwischen der Wetterlage und Rückenschmerzen hatte Steffens bereits vor zwei Jahren untersucht (Arthritis Care Res 2014; 66: 186772). Auch damals war das Ergebnis großenteils negativ ausgefallen, auch wenn sich für Böigkeit und Geschwindigkeit des Windes signifikante Assoziationen ergaben. Klinisch relevant waren die Effekte aber nicht. Viele Betroffene waren mit den Resultaten alles andere als einverstanden und gaben das in sozialen Netzen deutlich zu verstehen (u.a. hier bei "9 News" auf Facebook). Für die Forscher war das einer der Anlässe, sich dem Thema erneut zu widmen.

(rb)

Beilken Ket al. Acute Low Back Pain? Do Not Blame the Weather-A Case-Crossover Study. Pain Med 2016, online 15. Dezember; doi: 10.1093/pm/pnw126

\title{
Oskar-Medizinpreis 2016 zum Thema Pulmonale Hypertonie an Prof. Dr. Marius Hoeper
}

Berliner Stiftung Oskar-Helene-Heim vergibt den Oskar-Medizinpreis 2016, dotiert mit 50.000 Euro, auf dem Gebiet der Pneumologie für Forschungsarbeiten zur Weiterentwicklung von Diagnostik und Behandlung von Erkrankungen des Lungengefäßsystems

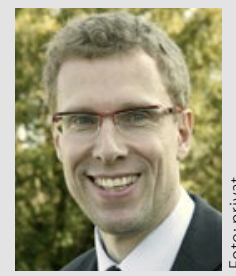

Prof. Dr. Marius Hoeper, Medizinische Hochschule Hannover, erhält den Oskar-Medizinpreis 2016. Der hochrangige Wissenschaftler wird für seine wegweisenden Forschungen zum Thema pulmonale Hypertonie ausgezeichnet.

Die pulmonale Hypertonie ist eine pathophysiologische Störung, die als Komplikation bei der MehrProf. Dr. zahl respiratorischer und kardiovaskulärer Krankheiten auftreten kann. Die Bezeichnung dient als Oberbegriff für verschiedenste Krankheitsbilder, die mit erhöhtem Blutdruck im Lungenkreislauf einhergehen. Es handelt sich um eine meist chronische Erkrankung, die im fortgeschrittenen Stadium als Folge von Rechtsherzversagen lebensbedrohlich sein kann. Voraussetzung für eine erfolgreiche Behandlung ist eine möglichst frühzeitige Diagnose. Da die Symptome bei der pulmonalen Hypertonie anfangs nicht spezifisch sind, wird die Diagnose häufig mit deutlicher Verzögerung gestellt und die Therapie demensprechend spät eingeleitet.
Mit der Verleihung an Prof. Hoeper würdigt die Stiftung die wissenschaftliche Qualität seiner 25-jährigen Forschungsarbeit, dokumentiert durch hochrangig publizierte Arbeiten und die Seniorautorschaft der aktuellen europäischen Leitlinie zur Diagnostik und Therapie der pulmonalen Hypertonie.

Das Preisgeld stellt die Stiftung für die Fortsetzung dieser Forschungen zur Verfügung.

Mitglieder der Jury für den Oskar-Medizinpreis 2016:

Prof. Dr. Berthold Jany, Missionsärztliche Klinik Würzburg

Prof. Dr. Horst Olschewski, Medizinische Universität Graz

Prof. Dr. Klaus F. Rabe, LungenClinik Großhansdorf

Prof. Dr. Silvia Ulrich Somaini, Universitätsspital Zürich

Prof. Dr. Helmut Teschler, Ruhrlandklinik Essen

Prof. Dr. Robert Loddenkemper, ehem. Lungenklinik Heckeshorn Berlin

Der Oskar-Medizinpreis 2016 wird auf dem Kongress der Deutschen Gesellschaft für Pneumologie und Beatmungsmedizin am 23. März 2017 in Stuttgart verliehen.

Stiftung Oskar-Helene Heim, www.stiftung-ohh.de, Geschäftsführer: Werner Ukas,

Kontakt:werner.ukas@stiftung-ohh.de

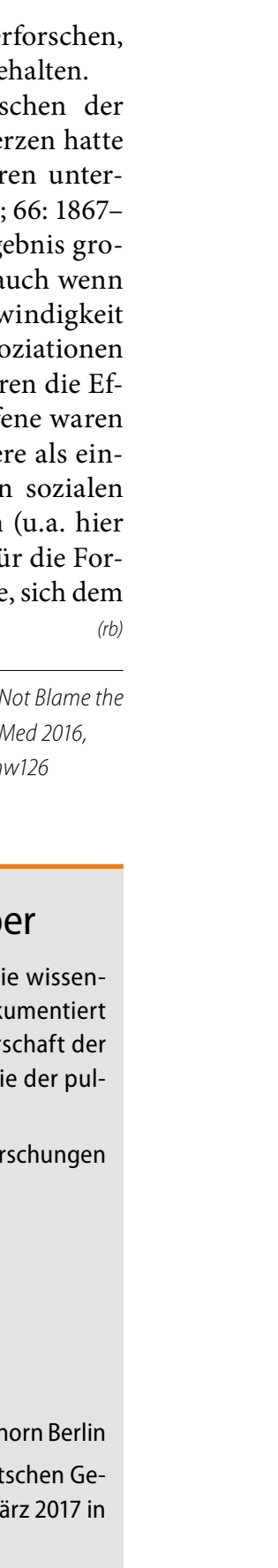

